\title{
Poisson Prediction of the loss of Teachers in High Schools
}

\author{
Li Yingqiu \\ College of Mathematics and \\ Computing Science, Changsha \\ University of Science and Technology \\ Changsha,China \\ liyq-2001@163.com
}

\author{
Sha Yongqiang \\ College of Mathematics and \\ Computing Science, Changsha \\ University of Science and Technology \\ Changsha,China \\ shayongqiang@126.com
}

\author{
ZhaoRenke \\ College of Mathematics and \\ Computing Science, Changsha \\ University of Science and Technology \\ Changsha,China \\ zhaorenke2006@126.com
}

\begin{abstract}
It is a key to the sustainable development and stability of colleges and universities education to predict the loss of college teachers. This paper analyzes the historic statistics and applies Chisquare test to do a hypothesis test on the distribution of intervals between two successive teachers' losses. It also sets up prediction models for the loss based on Poisson Process Theory. After some empirical analysis, it draws a conclusion and provides a new approach to forecast the staff turnover in colleges and universities.
\end{abstract}

Keywords-Exponential distribution; Poisson process; Prediction model; Data-fitting

\section{INTRODUCTION}

In today's society, human resources competition is the main competition. The status of human resources in high schools determines its overall comprehensive strength. University teachers are the subject of human resources and their team status determines the level of university teaching and research strength, thus human resource management should focus on construction of teachers. In recent years, many scholars dedicated to human resource management research, such as Markov process with transition probability matrix quantitatively predicting the allocation of teachers staff[1] [2], and this paper attempts to analyze the staff turnover of teacher system in a new perspective. We applied Poisson process theory to predict the loss of university teachers, and then we set up the prediction model in the loss of a system of university teachers' number in a certain period through the statistical analysis of historical data. Following the model, we can obtain the least upper bound of the number of random loss during $[0, t]$, the confidence level is high, which provides a reference that estimates the most actual number of teachers needed to be supplemented for the personnel management department.

\section{POISSON PREDICTION MODEL ON THE NUMBER OF SYSTEM STAFF TURNOVER}

Teacher wastage process is a counting process, when the time intervals of the loss of two successive staff are independent and exponentially distributed with the same parameters $\lambda$.In accordance with Poisson process theory we

This work was partially supported by the NNSF of China (Grant No.10771021 10471012), the Planned Science and Technology Project of Hunan Province (Grant No.2009fi3098) and Scientific Research Fund of Hunan Provincial Education Department (Grant No.09C113, 09C059, 08C120) can put the counting process as a Poisson process [3]. Then we established prediction model according to Poisson process for predicting the loss of teachers in college from the current time (denoted as 0 time) to the moment of time $t(t \geq 0)$.

\section{A. Time interval distribution of system turnover}

Statistics conditions of a college teacher turnover system are as follows.

Within the 124 weeks from March 20, 2006 to July 28, 2008, the loss of teachers in a college amounts to 130, the time intervals $\boldsymbol{\Delta}$ (weeks) of successive staff loss are as follows:

Table 1.Statistics for the time intervals $\boldsymbol{\Delta}$ of two successive staff loss

\begin{tabular}{c|cccccccc}
\hline $\begin{array}{c}\Delta \text { (weeks) } \\
\text { fall in the } \\
\text { range }\end{array}$ & {$[0,1]$} & $(1,2]$ & $(2,3]$ & $(3,4]$ & $(4,5]$ & $(5,6]$ & $(6,7]$ & $\begin{array}{c}(7,+ \\
\infty\end{array}$ \\
\hline $\begin{array}{c}\Delta \text { frequency } \\
\text { of occurrence }\end{array}$ & 78 & 30 & 12 & 5 & 3 & 1 & 1 & 0 \\
\hline
\end{tabular}

Let $\Delta_{l}$ denote time interval between $(l-1)$ th $(l=1,2, \cdots)$ staff turnover and $l$ th $(l=1,2, \cdots)$ staff turnover, which is obviously a continuous random variable. Considering the fact that it is independent from each other whether personnel in the system is still within the system in the future, then the time interval $\left\{\Delta_{l} \mid l \geq 1\right\}$ can be considered independent of each other.

Next, we apply Chi-square test to do a fit of exponential distribution on the distribution of time intervals, i.e. to test hypothesis $\mathrm{H}_{0}$ : the probability density of $\Delta_{l}$ is

$$
f(x)= \begin{cases}\lambda e^{-\lambda x}, & x>0 \\ 0, & x \leq 0\end{cases}
$$

Test process is as follows:

The estimated value of maximum likelihood of $\lambda$ is $\hat{\lambda}=1 / \bar{x}=130 / 124=1.0484$. If $\mathrm{H}_{0}$ is true, the estimate of the distribution function of $\Delta_{l}$ is: 


$$
\hat{F}(x)=\left\{\begin{array}{cc}
1-e^{-1.0484 x}, & x>0 \\
0 & , x \leq 0 .
\end{array}\right.
$$

Thus, table 2 shows the Chi-square test value table of the distribution of $\Delta_{l}$ by table 1 .

Table2. The value table of Chi-square test

\begin{tabular}{|c|c|c|c|c|c|c|}
\hline $\begin{array}{c}\text { Serial } \\
\text { number }\end{array}$ & Interval & $f_{i}$ & $\hat{p}_{i}$ & $n \hat{p}_{i}$ & $n \hat{p}_{i}-f_{i}$ & $\frac{\left(n \hat{p}_{i}-f_{i}\right)^{2}}{n \hat{p}_{i}}$ \\
\hline 1 & {$[0,1]$} & 78 & 0.614879 & 79.93431 & 1.934308 & 0.046807793 \\
\hline 2 & $(1,2]$ & 30 & 0.236803 & 30.78436 & 0.784357 & 0.019984702 \\
\hline 3 & $(2,3]$ & 12 & 0.091198 & 11.85569 & -0.14431 & 0.00175649 \\
\hline 4 & $(3,4]$ & 5 & 0.035122 & 4.565873 & & \\
\hline 5 & $(4,5]$ & 3 & 0.013526 & 1.758412 & & \\
\hline 6 & $(5,6]$ & 1 & 0.005209 & 0.677201 & -2.57436 & 0.892491756 \\
\hline 7 & $(6,7]$ & 1 & 0.002006 & 0.260804 & & \\
\hline 8 & $(7,+\infty)$ & 0 & 0.001257 & 0.163351 & & \\
\hline$\Sigma$ & & 130 & & & & $t^{2}=0.961040$ \\
\hline
\end{tabular}

Since $\chi_{0.05}^{2}(k-r-1)=\chi_{0.05}^{2}(2)=5.991>\chi^{2}=0.9610$, so $\quad$ it accepts hypothesis $\mathrm{H}_{0}$. Under the given confidence level of 0.95, it can be considered that the samples are from the exponential distribution with the parameters $\hat{\lambda}$, that is, the theoretical results are realistic. Therefore, it can be considered that: time intervals series of $\left\{\Delta_{l} \mid l \geq 1\right\}$ the loss of a college teacher system is independent and identically distributed, and for any $l, \Delta_{l}$ are subjected to the exponential distribution with parameters $\hat{\lambda}=1.0484$

$$
\Delta_{l} \sim \hat{f}(x)=\left\{\begin{array}{l}
1.0484 e^{-1.0484 x}, x>0 \\
0, \quad x \leq 0 .
\end{array}\right.
$$

\section{B. The distribution of the number of system turnover}

Let $N(t)$ denote the number of system turnover from the current time (denoted as 0 time) to the time $t(t \geq 0)$ the loss of teachers in colleges. $N(t)$ is the counting process with time continuous and state space $\{0,1,2,3, \cdots\}$.

By the definition of $\Delta_{l}$, the counting process $N(t)$ corresponds to the distance between points $\left\{\Delta_{l} \mid l \geq 1\right\}$. From 2.1 in the discussion results, we found that the distributions of time intervals $\Delta_{l}$ are the exponential distribution with parameters $\hat{\lambda}=1.0484$. And the time interval is independent of each other. Then by the Poisson process theory [4], $N(t)$ is a the Poisson process with parameters $\hat{\lambda}$. Then for any $t \geq 0$, we have

$$
P(N(t)=k)=\frac{(\hat{\lambda} t)^{k}}{k !} e^{-\hat{\lambda} t}, \quad k=0,1,2, \cdots,
$$

where $\hat{\lambda}=1.0484$

\section{Forecasting Model}

Let $0.95<\beta<0.99$, from equation (1), under the given confidence level $\beta$, the predicted value $s_{t, \beta}$ in the time interval $[0, t]$ denotes the number of system staff turnover in the college system, which is satisfied by

$$
s_{t, \beta}=\min _{s \in N}\left\{s: P(N(t) \leq s)=\sum_{k=0}^{s} \frac{(\hat{\lambda} t)^{k} e^{-\hat{\lambda} t}}{k !} \geq \beta\right\},
$$

where $\hat{\lambda}=1.0484$.

In the equation (2), predicted values $s_{t, \beta}$ means the minimum upper bound number of random loss during the time interval $[0, t]$, its confidence is greater than $\beta$. Or $s_{t, \beta}$ is an indicator to measure stability of teacher system in colleges.

\section{MODEL CHECKING}

\section{A. Calculation $s_{t, \beta}$ steps:}

Since $\sum_{k=0}^{S} \frac{(\hat{\lambda} t)^{k} e^{-\hat{\lambda} t}}{k !} \geq \beta$, i.e. , $\sum_{k=0}^{S} \frac{(\hat{\lambda} t)^{k}}{k !} \geq \beta e^{\hat{\lambda} t}$. Then calculated $s_{t, \beta}$ is as follows:

Step 1: Given $t, \beta, \hat{\lambda}$, calculated $\mu=\beta e^{\hat{\lambda} t}, \quad \gamma=\hat{\lambda} t ;$

Step 2: let $s=0, f_{1}=0$;

Step 3: Calculated $f_{s}=f_{s-1}+\frac{\gamma^{s}}{s !}+\sum_{k=0}^{s} \frac{(\hat{\lambda} t)^{k}}{k !}$;

Step 4: If $f_{s} \geq \mu$, let $s_{t}=s$, until calculated the end. If $f_{s}<\mu$, let $s=s+1$, turn to step 3 .

\section{B. Empirical analysis}

To further illustrate the validity and accuracy of the

\begin{tabular}{|c|c|c|c|c|c|}
\hline $\begin{array}{l}\text { Time range } \\
\text { (0,t(weeks)] }\end{array}$ & $\begin{array}{c}\text { Predicted } \\
\text { values }\end{array}$ & $\begin{array}{c}\text { Actual } \\
\text { loss } \\
\text { values }\end{array}$ & $\begin{array}{l}\text { Time range } \\
\text { (0,t(weeks)] }\end{array}$ & $\begin{array}{c}\text { Predicted } \\
\text { values }\end{array}$ & $\begin{array}{c}\text { Actual } \\
\text { loss } \\
\text { values }\end{array}$ \\
\hline$(0,1]$ & 3 & 2 & $(0,64]$ & 82 & 67 \\
\hline$(0,2]$ & 5 & 4 & $(0,68]$ & 86 & 70 \\
\hline$(0,4]$ & 8 & 9 & $(0,72]$ & 91 & 74 \\
\hline$(0,8]$ & 14 & 12 & $(0,76]$ & 95 & 76 \\
\hline$(0,12]$ & 19 & 16 & $(0,80]$ & 100 & 81 \\
\hline
\end{tabular}
model (2), the following to verify the model. According to calculated steps, we can obtain table 3 and figure 1 by MATLAB software. 


\begin{tabular}{l|ll|l|ll}
\hline$(0,16]$ & 24 & 20 & $(0,84]$ & 105 & 86 \\
$(0,20]$ & 29 & 23 & $(0,88]$ & 109 & 91 \\
$(0,24]$ & 34 & 27 & $(0,92]$ & 114 & 95 \\
$(0,28]$ & 39 & 32 & $(0,96]$ & 119 & 98 \\
$(0,32]$ & 44 & 36 & $(0,100]$ & 123 & 102 \\
$(0,36]$ & 49 & 41 & $(0,104]$ & 128 & 105 \\
$(0,40]$ & 54 & 44 & $(0,108]$ & 132 & 110 \\
$(0,44]$ & 58 & 47 & $(0,112]$ & 137 & 116 \\
$(0,48]$ & 63 & 52 & $(0,116]$ & 141 & 121 \\
$(0,52]$ & 68 & 55 & $(0,120]$ & 146 & 126 \\
$(0,56]$ & 72 & 58 & $(0,124]$ & 150 & 129 \\
$(0,60]$ & 77 & 62 & & & \\
\hline
\end{tabular}

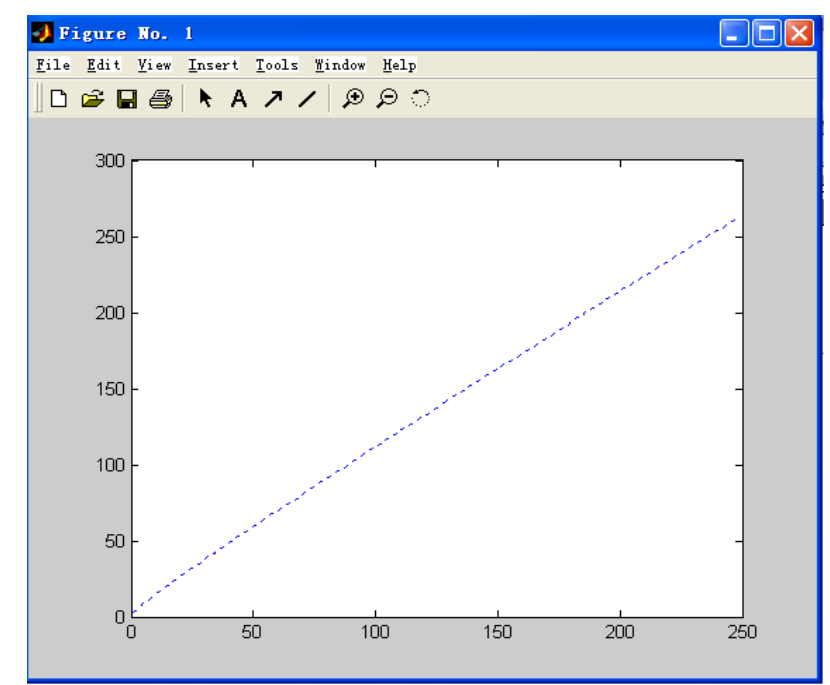

Fig 1.the curve of the prediction values $s_{t, \beta}$ (where $\beta=0.96, \hat{\lambda}=1.0484$ ).

(X-axis represents time, $\mathrm{Y}$-axis represents optimal predictive value)

Table 3 shows the actual values and prediction values of introducing to the system of teachers in 2007 and 2008. According to the table 3, it shows that the relative error between the number of actual loss and prediction values is less than 5\%, generally in correspond with the actual situation, so the model is accurate and effective. By analyzing figure 1 , we also find that the predicted values $s_{t, \beta}$ is a linear function of the time $t$, and the probability of the actual value curve below the forecast value is more than $95 \%$, and then compare them, under the given confidence level we can obtain the extent of its stability, and the greater confidence, the better stability.

\section{CONCLUSIONS}

According to the system staff turnover, we can establish the prediction model for teachers system using Poisson process theory analysis for the first time, and validate the model accuracy using the school's historical data. The results show that we can set up prediction models for the loss based on Poisson Process Theory and apply the model to predict the loss of staff, and then estimate the actual supplement staff and add the actual number of teachers, which provides a new scientific evaluation criterion for measuring the stability of the system staff turnover.

\section{REFERENCES}

[1] Zeng M F. Application for the building of teachers in high schools based on state transition matrix method[J]. J Chifeng College(Natural Science Edition),2006,04-0105-02.

[2] Lin S W, Wang Y S. A study on the model to predict the faculty change at colleges and universities[J]. J Fujian Agri Fore Univ(phil Sci), 2005,8 (2):53-55.

[3] Sun R H. Stochastic processes and its application[M]. Beijing: Tsinghua University Press, 2004.

[4] Yu J H, Yang W Q. Multivariate Statistical Analysis and Application [M]. Guangzhou: Zhongshan University Press, 2005.

[5] Li Y Q, Feng R L, Peng Z H. The quantitative research of the income distribution gap based on regression analysis. J Changsha Uni Sci Tech(Social Science), 2006,21(3):60-66.

[6] Li Y Q, Liu W, Chen W F. Differences research in the insurance industry of regional in cluster analysis perspective Analysis of pool data based on all over the city of Hunan province. Times Finance, 2009,(1):117-119.

[7] Li Y Q, Liu Z C, Peng Z H. Decision-making on Investment Capacity under Uncertainty. Operations Research Transactions, 2008,12(2):121128.

[8] Peng Z H, Feng R L, Li Y Q. Urban-Rural Inequality in Hunan and Its Influential Factors. J Changsha Uni Sci Tech(Social Science), 2008,23 (1):110-1 